\title{
Forecasting of Polymer Concrete Thermo-Moisture Creep
}

\author{
J. Hristova, R. Bares \\ Czechoslovak Inst. of Sciences, Inst. of Theoretical Applied Mechanics \\ Vysehradska 49, Prague 2, 12849, Czechoslovakia
}

\begin{abstract}
The possibilities of forecasting the thermo-moisture creep of polyester polymer concrete under compressive load with the help of time-temperature-moisture analogy (superposition) are presented. The forecasting is carried out on the basis of short-term (10 hours) creep experiments of polymer concrete samples at constant values of temperature and moisture content. Two major problems have been solved on the basis of the experimental data: a model of creep behaviour at normal temperature for a considerable period of time, exceeding by several orders the short-term experiment, has been created and creep processes at a set temperature in the investigated range have been predicted with a sufficient degree of accuracy.
\end{abstract}

\section{INTRODUCTION}

Investigations of the complex influence of temperature and water on polymer concrete creep (thermo-moisture creep) are of interest both from theoretical and practical points of view, since many polymer concrete constructions are subjected to the parallel effects of mechanical loading, elevated temperature and liquid medium (technological or waste liquids, etc.).

It has been established from experimental creep data that temperature and liquids (water in particular) manifest themselves as factors accelerating relaxation processes. This enables the application of the analogy (superposition) method to forecast polymer concrete creep under different temperature-moisture conditions. Thus, with a definite degree of approximation, it is possible to foresee the behaviour of the material under service conditions.
A very important point of prognostication is the establishment of the accuracy and reliability of the prognosis. This is achieved by juxtaposition of the forecasted values with the data obtained from a long-term control experiment, using statistical methods for the assessment of coincidence.

\section{MATERIALS AND EXPERIMENTAL METHODS}

The investigated composite commonly known as polymer concrete (PC), is constituted from a polymer binder (usually a thermoset resin) and rocky mineral grains of different sizes (filler, aggregate) and thus combines some characteristics features of both plastics and concrete. In spite of the fact that this material is mostly comprised of grains of various size and elastic properties (the polymer binder is only $8-15 \%$ ), it displays a typical relationship of mechanical properties with temperature and time of a polymer material. To a first approximation, $\mathrm{PC}$ can be considered as composed of two basic structural elements: matrix and aggregate. The matrix is formed by the thermoset resin and the filler, while the aggregate is a mixture of fractions with different grain size which fulfil the requirement for a minimum volume of cavities. The carrier of the viscoelastic properties of PC is the matrix.

The study of the complex influence of temperature (t) and water $(w)$ is made by investigation of creep of specimens with different water content under isothermal conditions. PC prisms containing a definite quantity of liquid, permeated by exposure to a fluid medium, are used. Before creep tests the samples are drawn out of the water and covered by an impermeable layer to keep the amount of permeated fluid constant during the creep experiment. Because of the density of the polymer 
concrete investigated, the limits to which content can vary are up to $\mathrm{w}=0.98 \%$ (reached after exposure in water for a period longer than one year). The influence of temperature is investigated under isothermal conditions at $\mathrm{T}=20-50^{\circ} \mathrm{C}$ corresponding to the glassy state of the thermoset polymer and of the composite material.

Experiments have been carried out under compressive loading in the linear range of creep. From the mean values of creep deformation, the constant stress has been calculated and has been used for results analysis. Experiments may be divided into two groups; basic and control. The first continue for 10 hours and their purpose is to establish the relationship between creep deformation and the factors investigated as well as the applicability of both the rheologically simple body model and the analogy method for PC. The second continue for at least 100 days and are used for the assessment of the real possibillities of predicting creep behaviour by means of time-temperature-moisture analogy (TTMA).

\section{THE METHOD OF TIME-TEMPERATURE-MOISTURE ANALOG Y APPLIED TO PC}

A suitable approach for prognostication rheological properties of polymer materials is the analogy (superposition) method. It is based on the use of factors accelerating relaxation processes (temperature, moisture content, etc.). The problem of rheological behaviour forecasting by means of the analogy method, the accelerating factors being known, is solved by experimental determination of the scale function of time (termed also a shift [reduction] function). For this purpose, short-term creep experiments are conducted at constant values of the accelerating factors. The master curve and the transformed (reduced) time are obtained from the shift function.

The analogy method in different forms has been propounded by Leaderman, Tobolsky, Andrews, Ferry, Williams, Landel, Urzumtsev, Maximov, etc. The validity of the analogy method for PC was confirmed experimentally by Hristova / 1 .

The case under consideration in the present work is PC behaviour under the action of two accelerating factors, elevated temperature and higher water content, so that TTMA is applicable. The cited data concern polyester PC (PPC). The values of the accelerating factors are as follows: $w=0.01,0.53$ and $0.98 \%$ (by mass) and $\mathrm{T}=20,3040$ and $50^{\circ} \mathrm{C}$.

TTMA is valid for polymer materials when they preserve their rheologically simple behaviour under the simultaneous action of the two factors accelerasting relaxation processes: $T$ and $w$.

The temperature-moisture-rheologically simple behaviour of PPC is established from the affinic similarity of creep curves obtained for a given w at several constant values of temperature (i.e., from the thermorheologically simple behaviour at a fixed, but different $w$ ) and for a given $T$ at several fixed values of water content (i.e., from the moisture-rheologically simple behaviour at a constant, but different $\mathrm{T}$ ).

Analysis of experimental results shows that if timetemperature analogy is applied to test specimens with fixed, but different magnitude of $w$ the values of the time-temperature shift function $a_{T}(T)$ for a single w are close to one another and may be assumed to coincide (Figs. 1a, b). On the other hand, if TMA is applied to specimens under isothermal conditions at different $T$, the values of the time-moisture shift function $a_{w}(w)$ at a particular value of $\mathrm{T}$ are also very close or coincide (Figs. 1c, d). On the basis of the above mentioned facts, it is established that when the two accelerating factors $w$ and $T$ act simultaneously, their influence on the rheological behaviour of PC is independent, i.e., the time-temperature-moisture shift function $\mathrm{a}_{\mathrm{T}}(\mathrm{T}, \mathrm{w})=$ $a_{T}(T) \cdot a_{w}(w)$ so that $\ln a_{T w}=\ln a_{T}(T)+\ln a_{w}(w)$.

The rheologically simple behaviour of polymer concrete under the influence of variation of $T$ and $w$ and their combination enables the creation of one combined master curve (Fig. $2 b$ ) and one combined shift function ${ }^{a_{T w}}$ (T, w) (Fig. 2c).

This is achieved by shifting all experimental data (given in Fig. 2a in the coordinates $J-\ln t$ ) for the corresponding $\mathrm{T}$ and $\mathrm{w}$ along the abscissus at a distance $\ln \mathrm{a}_{\mathrm{w}}$ ( $\left.\mathrm{T}, \mathrm{w}\right)$. Such an approach employs the simultaneous influence of the two factors ( $T$ and $w$ ) accelerating the relaxation processes in polymer concrete by means of the analogy method. Thus, the use of the time-temperature-moisture analogy leads to an extension of the ways to forecast and to describe creep behaviour.

\section{FORECASTING OF PPC CREEP}

The determination of the shift function and the master curve from the experimental data is the first step in this investigation. The second step is of great practical importance, the prediction of the rheological behaviour of PC. At constant values of the accelerating factors, this can be performed directly from the master curve and the shift function. In order to forecast creep behaviour it is 


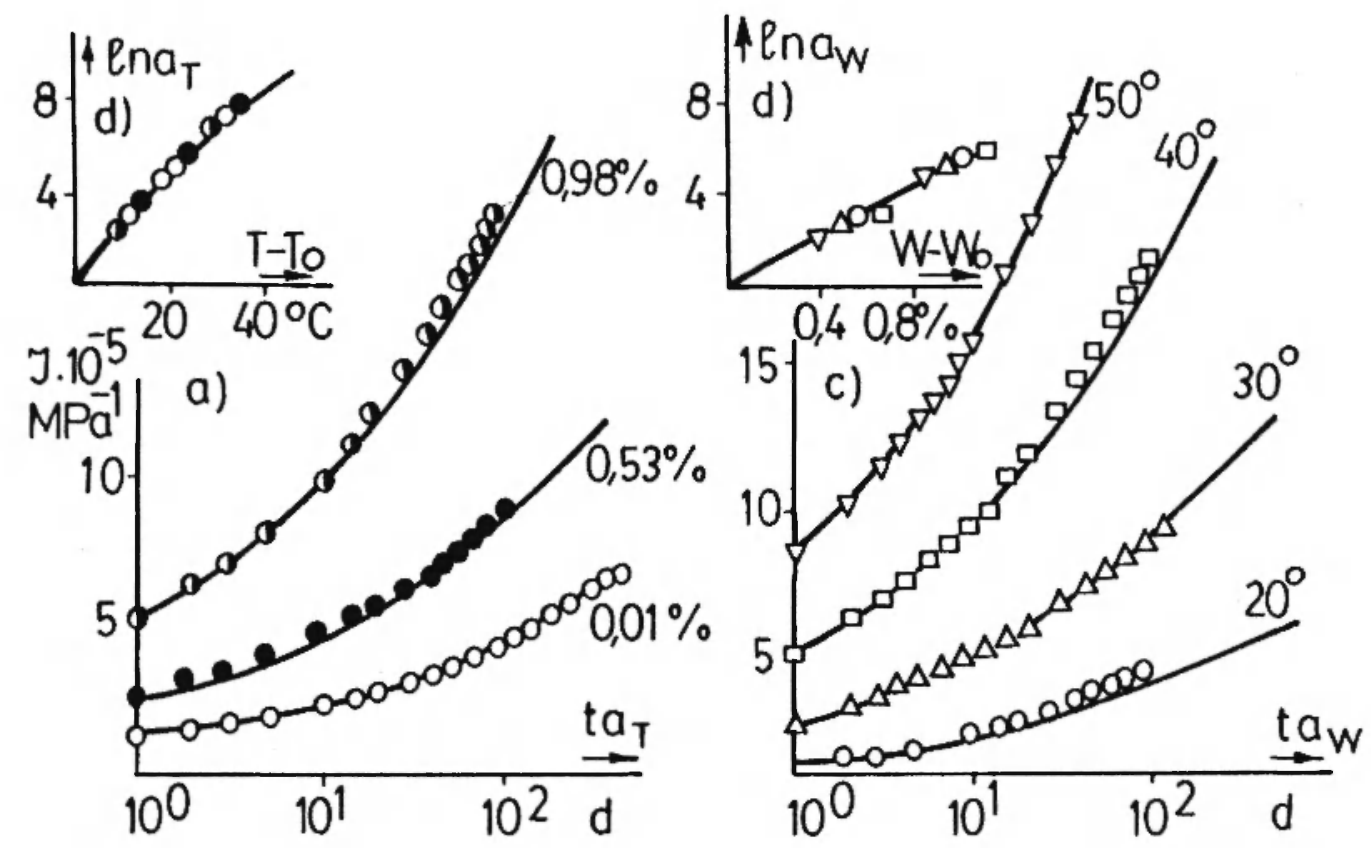

Fig. 1: Master curves $(a, c)$ and shift functions $(b, d)$, obtained by means of time-temperature analogy $(a, b)$ and of time-moisture analogy $(c, d)$; experimental values from control tests are shown.

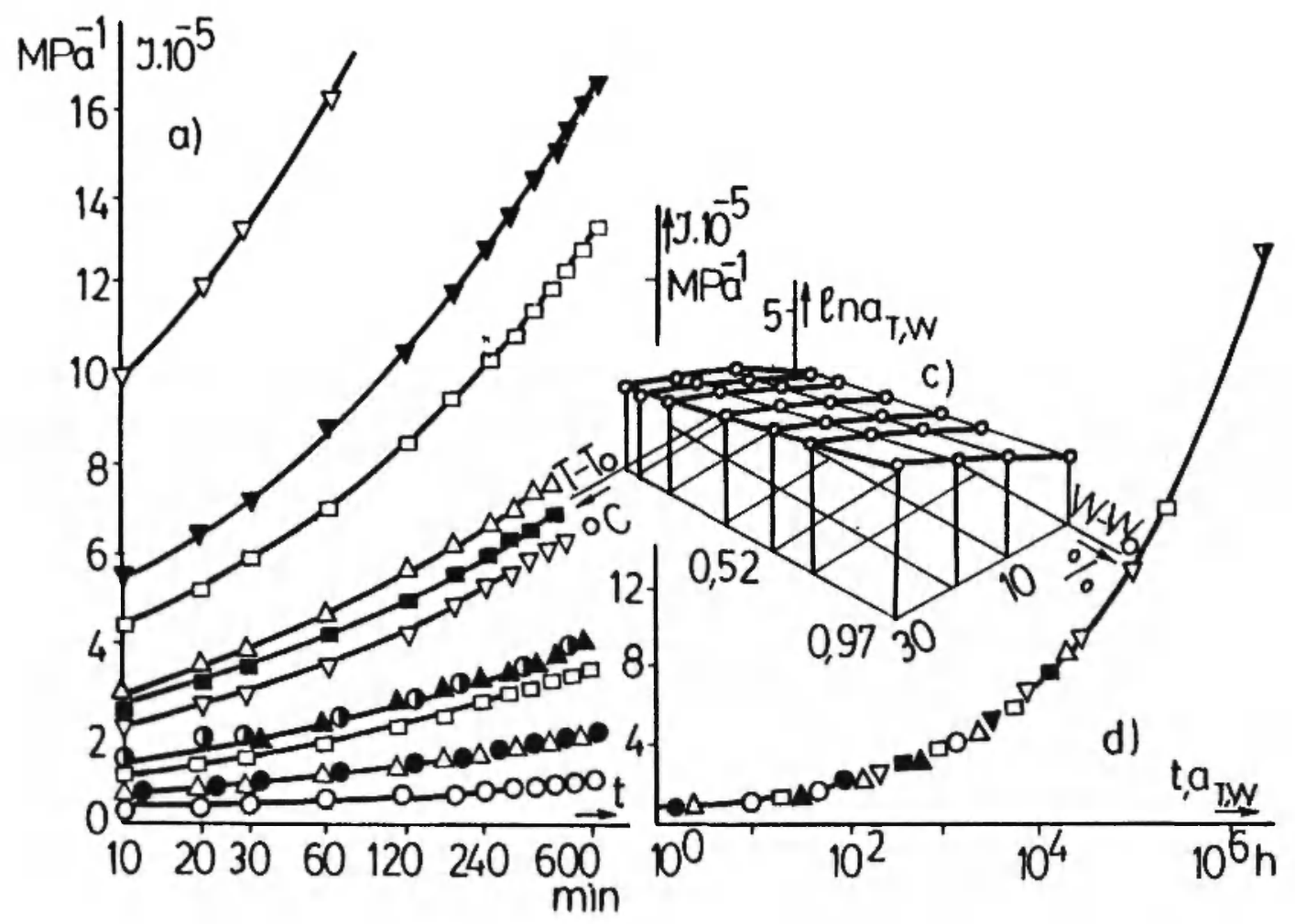

Fig. 2: Compliance curves for the investigated $T$ and $w$, obtained from short-term experiments (a), master curve (b) and shift function (c) , constructed by means of TTMA $\left(T_{0}=20^{\circ} \mathrm{C}, w_{0}=0.01 \%\right)$. 
necessary to know the shift function $a_{T}(T)$ and the master curve for some of the viscoelastic characteristics of PC, for example, the compliance J, or the creep modulus. The creep compliance $\mathbf{J}$ is considered in such a case. The prognosticated compliance values can be used to forecast creep under different stresses within the linear range.

The time interval of the prognosis depends on the length of the time scale of the master curve and on the properties of PC. The length of the time scale of the master curve is related to the values of $T$ and $w$ and to the duration of the short-term experiment. The difference between the length of the master curve and the duration of the short-term experiment is many orders of magnitude (in this case more than five orders).

To predict $\mathrm{J}$ at the reference $\mathrm{T}_{\mathrm{o}}$ and $\mathrm{w}_{0}$, the master curve may be used. The time interval over which the forecast is correct is determined by comparison with the results of the control experiment (Fig. 3).

The time interval of the prognosis diminished with increasing values of $T$ and $w$, for the range in which the function $a_{T_{w}}$ is defined, as illustrated by Figs. 4-6.

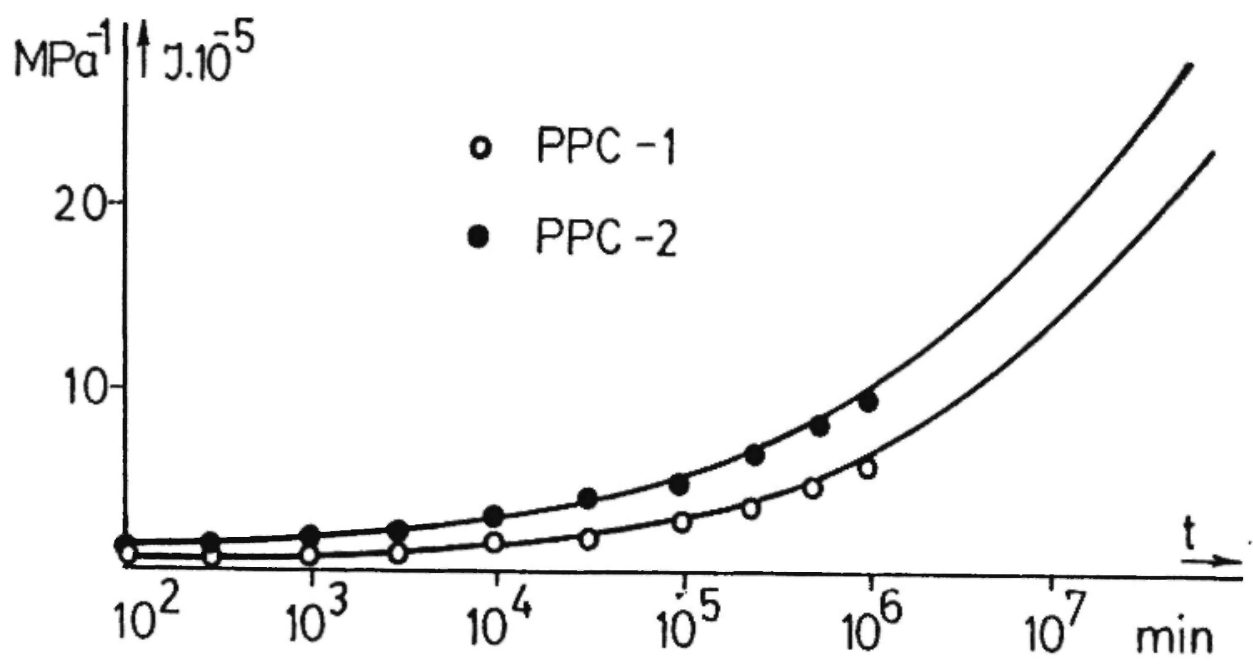

Fig. 3: Compliance curves at $\mathrm{T}=20^{\circ} \mathrm{C}$ and $\mathrm{w}=0.01 \%$, forecast by TTMA (master curves) for two compositions of PPC, compared with experimental values obtained from control tests.

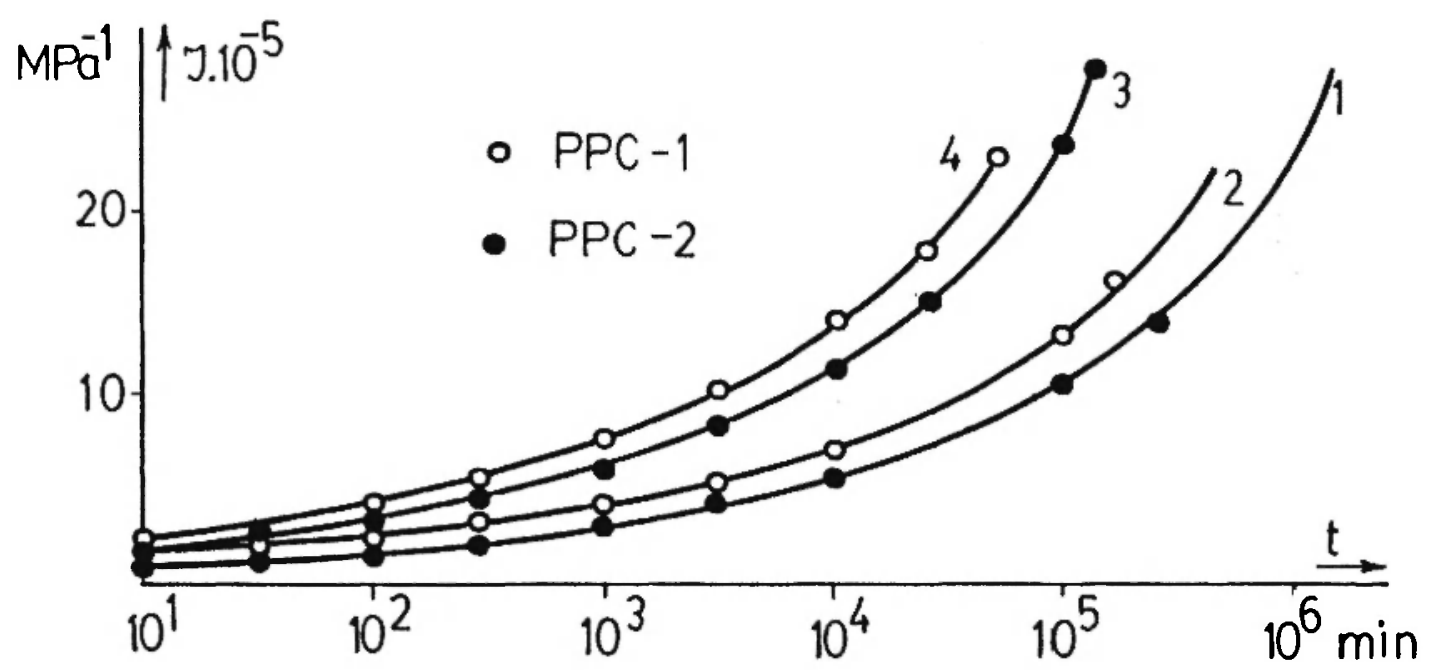

Fig. 4: Compliance curves at $w=0.01 \%$ and $T=30 \hat{} \mathrm{C}(1) 37 \hat{C}(2), 40 \hat{C}$ (3), 50 $\hat{C}$ (4), forecasted by TTMA for two compositions of PPC, compared with experimental values obtained from control tests. 


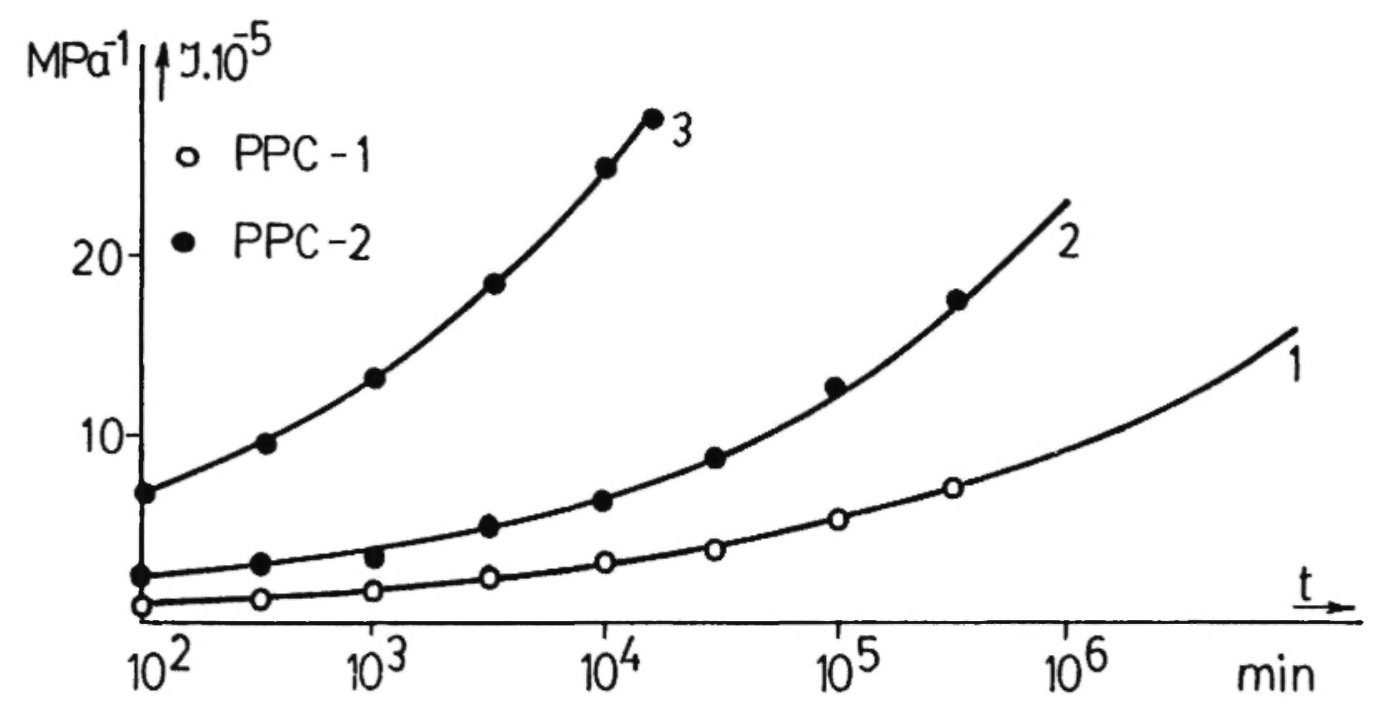

Fig. 5: Compliance curves at $T=20^{\circ} \mathrm{C}$ and $w=0.16 \%(1), 0.21 \%(2), 0.70 \%$ (3), forecasted by TTMA for two compositions of PPC, compared with experimental values obtained from control tests.

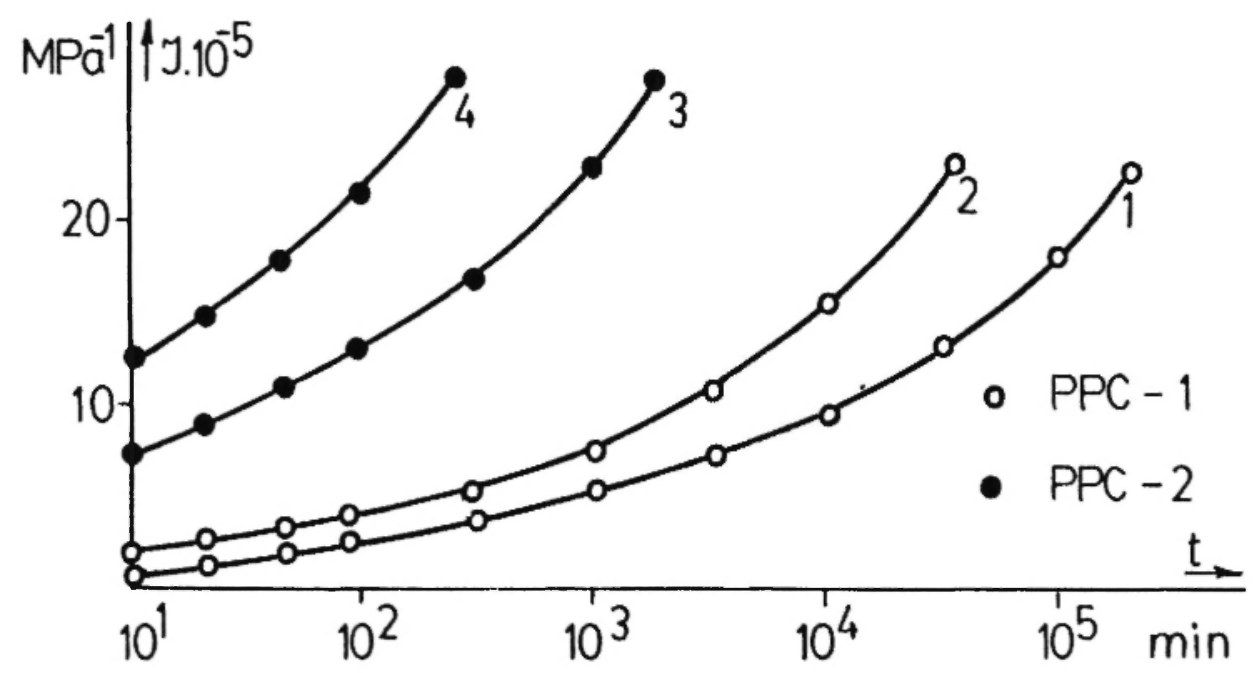

Fig. 6: Compliance curves at $\mathrm{T}=30^{\circ} \mathrm{C}$ and $\mathrm{w}=0.13 \%(1), \mathrm{T}=40^{\circ} \mathrm{C}$ and $\mathrm{w}=0.36 \%(2), \mathrm{T}=40^{\circ} \mathrm{C}$ and $\mathrm{w}=0.40 \%(3), \mathrm{T}=50^{\circ} \mathrm{C}$ and $\mathrm{w}$ $=0.40 \%(4)$, forecasted by TTMA for two compositions of PPC, compared with experimental values obtained from control test..

The time scale of prognostication may be prolonged by increasing either the duration of the short-term experiment or the values of the accelerating factors $\mathrm{T}$ and $w$.

The coincidence between the prediction and the experiment over the indicated time interval (Figs. 3-6) is quite satisfactory. The coefficient of variation does not exceed $10 \%$. This confirms the ability of forecasting PC creep behaviour by means of TTMA.

Another approach revealing greater possibilities since it allows creep prognostication and description under non-stationary temperature-moisture conditions, is the 
application of the linear relationships of viscoelasticity. In this way, a more concise expression of thermal-moisture creep effects could be achieved.

\section{CONCLUSIONS}

TTMA offers a possibility of forecasting PC creep behaviour at a reference temperature $T_{0}$ and water content $w_{o}$ and to prognosticate the thermo-moisture creep at different values of $T$ and $w$ within the range of a known time-temperature-moisture shift function.

The short-term experiments, which are the basis of the application of TTMA, may be carried out under specially selected conditions, in order to obtain a forecast for a practically useful time interval.

\section{REFERENCE}

1. HRISTOVA, J, Proc. IX Int. Congress on Rheology, 3: 409416 (1984). 